\title{
Latinoamérica ante las transformaciones del Orden Monetario y Financiero Mundial
}

Latin America beforehand the transformations of Monetary and Financial World Order

Paulo Humberto Leal Villegas*

\section{Resumen}

El presente artículo tiene como objetivo analizar las implicaciones que ha traído para la región de América Latina las transformaciones en la economía mundial, en particular los referentes al sector monetario y financiero y a las variaciones en los precios de las commodities. Para realizar este análisis, se han seleccionado las economías de cuatro países latinoamericanos por considerarse las cuatro principales economías de la región:Argentina, Brasil, Colombia y México. La hipótesis de este artículo es que el canal de transmisión de la inestabilidad financiera y monetaria a nivel global hacia Latinoamérica cambia de país a país dependiendo de la estructura productiva y de la forma de inserción a la economía mundial. Así mientras en algunos casos la caída del precio internacional del petróleo es fundamental, en otro la sobreexposición al comportamiento de la política monetaria estadounidense es un factor central, mientras que la inestabilidad financiera y política interna juega un papel central en otro caso particular.

\section{Palabras clave:}

- Orden económico internacional

- Integración económica

- Mercados financieros y macroeconomía

\begin{abstract}
This article aims to analyze the implications brought to Latin America transformations in the world economy, in particular those relating to monetary and financial sector and changes in commodity prices. To perform this analysis, we have selected economies by four Latin American countries considered the four major economies in the region: Argentina, Brazil, Colombia and Mexico. The hypothesis of this paper is that the transmission channel of monetary and financial instability globally to Latin America varies from country to country depending on the production structure and the way of integration into the world economy. So while in some cases the fall in international oil prices is critical, another over exposure to the behavior of US monetary policy is a central factor, while financial instability and domestic policy plays a central role in one particular case.
\end{abstract}

Keywords:

- International Economic Order

- Economic Integration

- $\quad$ Financial Markets and Macroeconomics

JEL: F02, F15, E44

\section{Introducción}

Las principales monedas latinoamericanas han sufrido un proceso de devaluación con respecto del dólar durante los últimos meses de año 2014 y el primer semestre de 2015. Las explicaciones de este fenómeno son muy variadas, tienen que ver con la estructura productiva de cada país y de cómo se insertan en la economía mundial en un entrono de bajos precios de las commodities y de alta volatilidad financiera. Con el objetivo de avanzar en el análisis del

*Profesor de Tiempo Completo de la Facultad de Economía de la UNAM, Doctor en Economía por el IIEC-UNAM y Miembro del Proglocode. Este artículo es una versión resumida de la ponencia presentada 


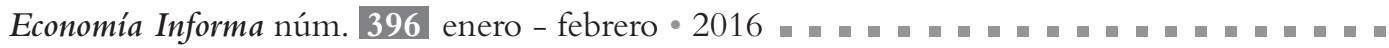

impacto que ha tenido el entorno económico mundial en la región latinoamericana, se seleccionaron las monedas de las cuatro principales economías latinoamericanas: el Real Brasileño, el Peso Mexicano, el Peso Argentino y el Peso Colombiano.

Este artículo está compuesto de dos partes y una conclusión que presenta escenarios posibles. En la primera parte se analiza el entrono financiero mundial, en la segunda parte se realiza un análisis particularizado de cada uno de los países latinoamericanos en la cual se avanza en proponer una explicación particular del proceso de devaluación monetaria y de las perspectivas de cada nación, finalmente en las conclusiones se presentan los nexos entre la economía mundial y las economías latinoamericanas y se presentan posibles escenarios a futuro.

\section{El entorno económico y financiero mundial}

El entorno monetario y financiero mundial se encuentra en un proceso continuo de transformación y ajuste. El cual es el producto de un entorno conflictivo de juego suma cero en el que un país gana lo que otro pierde, por ello cada nación busca defenderse lo mejor posible de la especulación global. El mejor ejemplo de esto es la transformación que ha sufrido la política monetaria de Estados Unidos, a raíz de la crisis de 2008-2009. La política monetaria de Estados Unidos se ha tornado altamente líquida, la más líquida de la historia (ver gráfica 1).

La inestabilidad financiera mundial es el resultado de un largo proceso de descomposición de la economía real de Estados Unidos y de su respuesta mediante el dominio que ejerce sobre el entorno monetario y financiero mundial al tener la moneda de reserva mundial, el dólar (Leal 2015). Esta respuesta resuelve en el corto plazo la problemática estadounidense, pero al mismo tiempo logra que el resto del mundo financiemos sus carencias mediante la demanda de dólares.

En este orden de ideas observamos que el origen del incremento en la liquidez coincide con el inicio del déficit de cuenta corriente de Estados Unidos, esto es ante la complejidad por satisfacer su demanda interna, Estados Unidos ha recurrido a la emisión monetaria para poder importar los montos de bienes de capital y bienes de consumo que requiere su economía (ver gráfica 2). 


\section{Gráfica I}

\section{Base Monetaria de Estados Unidos 1959-2015}

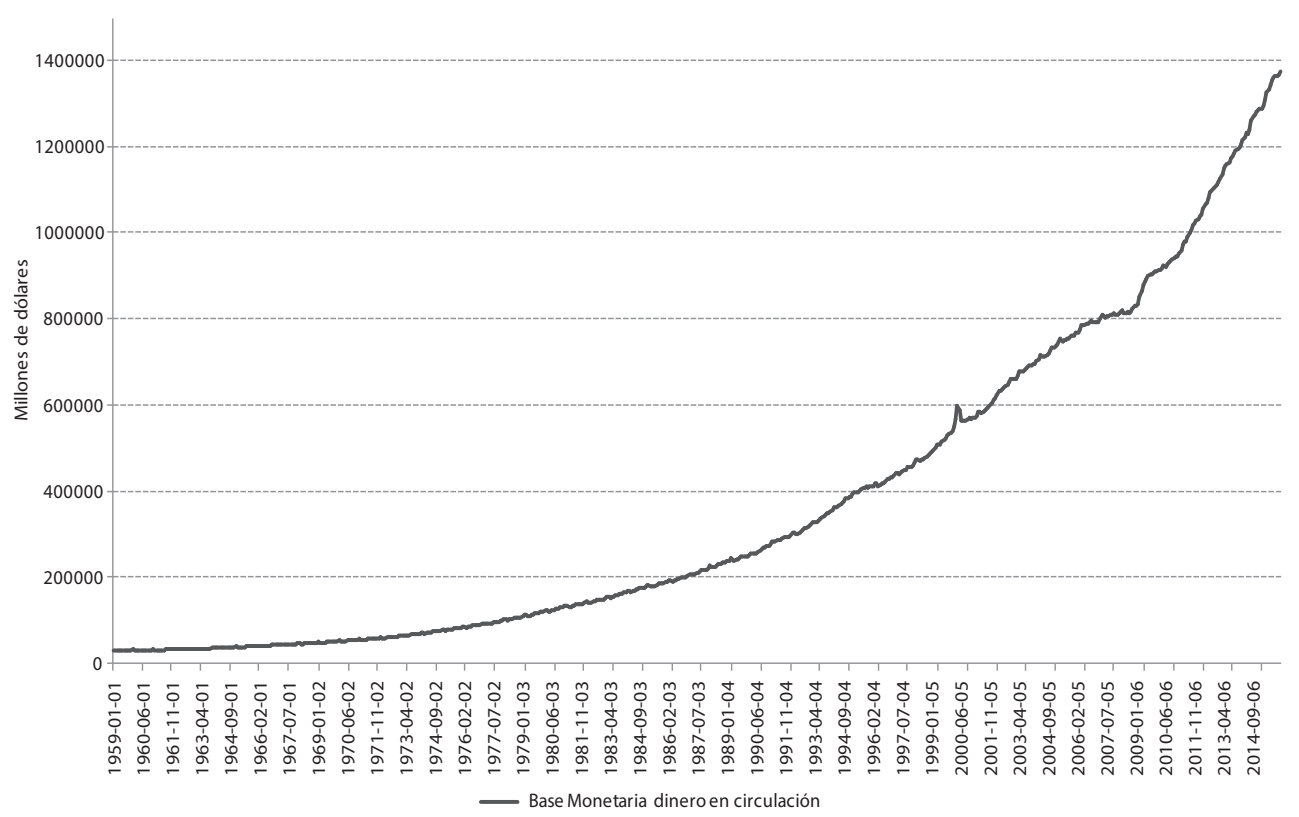

Fuente: Board of Governors of the Federal Reserve System (USA)

Estados Unidos puede vivir con un creciente déficit de cuenta corriente porque es el dueño del dólar, así mediante las reservas internacionales todos los países estamos financiando su déficit de cuenta corriente y la liquidez mundial aumenta.

Ante esta situación, la cantidad de dólares en circulación no está respaldada por el PIB de la economía más fuerte en términos competitivos, por ello la tenencia de dólares fuera de Estados Unidos crece buscando espacios de rentabilidad, pero las tasas de interés están por los suelos. Por lo que el dinero en circulación se encuentra en los mercados de bonos corporativos de renta fija, lo que ha generado un mercado mucho más grande pero también más frágil. Este mercado de renta fija de Estados Unidos representa uno de los mayores mercados financieros del mundo 39.5 miles de millones de dólares en circulación a mediados de 2015, según la Asociación de la Industria de Valores y Mercados Financieros (Sifma, por sus siglas en inglés).

En este contexto de alta liquidez y volatilidad, los mercados de renta fija, asociados desde hace tiempo con mayor seguridad y previsibilidad, parecen más vulnerables que nunca a cambios de precios e interrupciones de operaciones que podrían desbordarse y amenazar el financiamiento de empresas y consumidores (Barr 2015). 
El riesgo financiero mundial se agudiza si observamos el diferencial entre los rendimientos de los bonos corporativos y los bonos del tesoro en Estados Unidos, a este diferencial lo conocemos como spread. El aumento del spread implica que ante la seguridad absoluta con la que cuentan los bonos gubernamentales, el público requiere de un mayor premio o rendimiento por arriesgarse en la compra de bonos corporativos, este comportamiento del mercado genera fuertes presiones sobre las empresas lo que puede llevarlas a un gran nivel de apalancamiento y finalmente a la ruptura de la cadena de pagos (Cherney 2015).

\section{Gráfica 2}

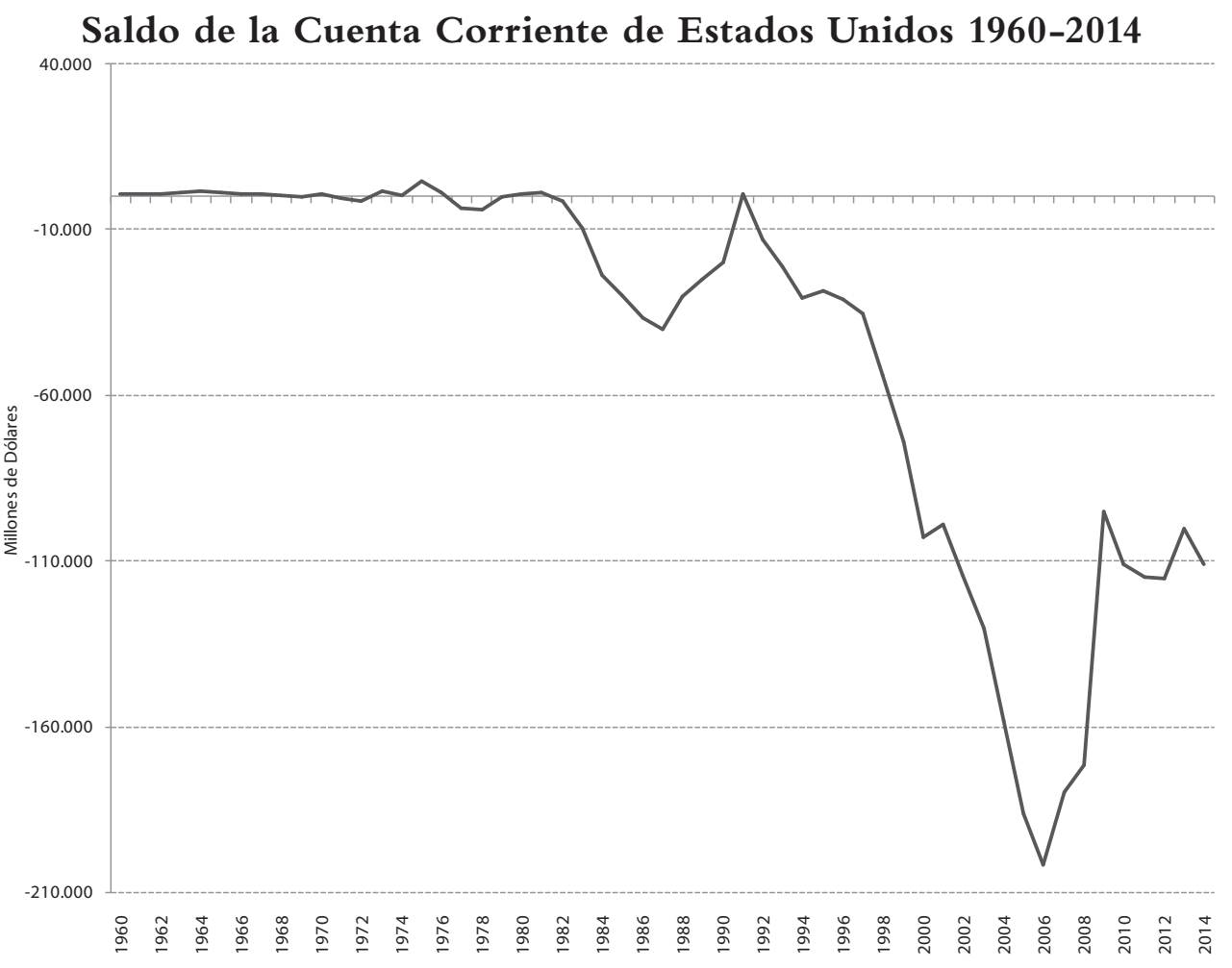

Fuente: Bureau of Economic Analysis USA.

Para redondear aún más el entorno de gran inestabilidad financiera, se debe mencionar que los bonos corporativos de los países latinoamericanos también han mostrado señales de comportamientos a la baja ante la caída de los precios de las materias primas (ver sección siguiente). 
Ante este contexto de alta inestabilidad, el sector financiero ha buscado "monedas puerto seguro" para combatir la inestabilidad: Yen, Euro, Franco, Libra y Dólar. Pero la zona Euro está amenazada por la crisis de deuda en Grecia, España, Portugal e Italia. Por lo que el Euro se ha devaluado de manera importante frente al dólar. El Franco suizo no tiene el sustento productivo para recibir una parte tan importante de la liquidez mundial por lo que el gobierno suizo reiteradamente interviene en el mercado de cambios para evitar que la entrada de capitales financieros aprecie demasiado su moneda y genere pérdida de la competitividad en su sector exportador que no es muy grande, el Yen japonés no ha logrado convertirse en una moneda que gane liderazgo a nivel mundial y en el caso de la Libra Esterlina también se ha depreciado con respecto del dólar.

De acuerdo con Cherney (2015) esta política de aumento de liquidez (QE) conduce a la economía de Estados Unidos a un callejón sin salida, porque el aumento del spread ha derivado en el pasado en una importante quiebra de empresas. Además, las aseguradoras están sufriendo ante las bajísimas tasas de interés, porque depositan los montos recibidos por concepto de pólizas en instrumentos bancarios o en bonos del tesoro con tasas de menos de 1\%. Por lo que de mantenerse mucho tiempo más la tasa de interés en esos niveles se avecina un nuevo problema financiero, ahora en el mercado de seguros.

En resumen la QE ha generado mucha inestabilidad en el plano financiero. Sin embargo, ese aumento de liquidez no ha redundado en un incremento suficiente de la inversión productiva. Si bien la tasa de interés en Estados Unidos ha caído a niveles cercanos al $0 \%$ ( $0.2 \%$ en este año ver gráfica 3$)$ y con ello ha arrastrado a las tasas de interés de los principales países, esto no ha redundado en un incremento importante de la inversión productiva en Estados Unidos. 


\section{Gráfica 3}

Tasa de interés de los Bonos del Tesoro Estados Unidos 1940-Junio 2015

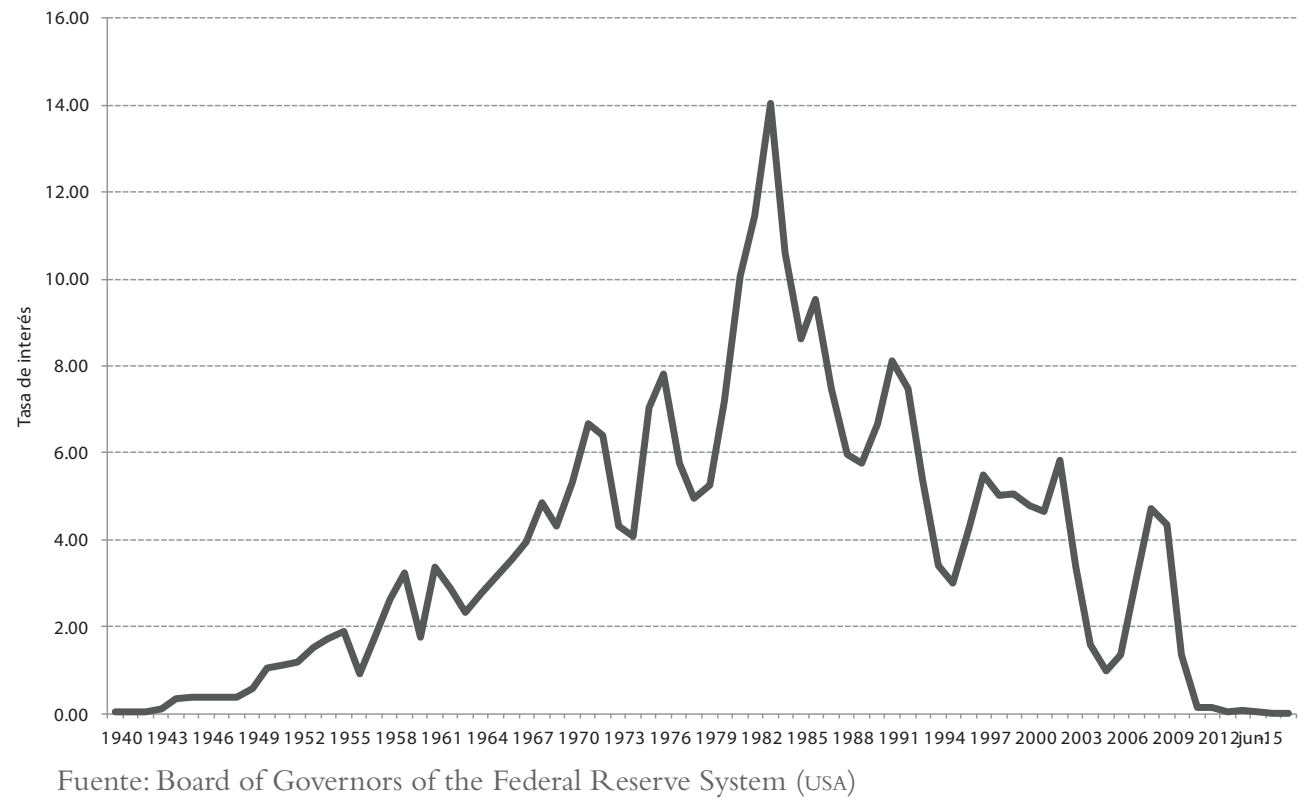

La prueba de esto es el nivel de desempleo en Estados Unidos que se mantiene por encima del 5\%, ante este escenario de baja rentabilidad financiera, (por las tasa de interés) y bajos niveles de ganancia en el sector real de Estados Unidos, el sector financiero ha buscado rentabilidad no solo en loe mercados de bonos, sino especulando con monedas a lo largo de prácticamente todo el mundo, en este contexto América Latina no ha sido la excepción como veremos a continuación.

\section{Impacto en el mercado cambiario de países seleccionados de Latinoamérica}

\section{I Argentina}

En el caso de Argentina la devaluación que se observa en el cuadro 1 del orden de 129\% entre enero de 2011 y junio de 2015 no es producto de la inestabilidad financiera internacional desatada a fines del año pasado ante la probabilidad de incremento en la tasa de interés de Estados Unidos. Sino más bien responde a la estructura financiera interna de ese país. 
Por otro lado, su comercio exterior tampoco ha sufrido los embates de la caída de los precios internacionales de las materias primas con la intensidad de los demás países latinoamericanos. La estructura del comercio internacional de Argentina los coloca en una situación ambigua de fortalezas y debilidades.

En el análisis detallado de su comercio exterior observamos que los principales productos para la exportación en Argentina son: la soya y sus derivados, el petróleo, el gas, automóviles, maíz y trigo (CIA 2015). Es decir básicamente materias primas, aunque ha bajado el precio de la soya (21\% de enero de 2014 a junio de 2015 World Bank), la caída ha sido menor que el precio de otras materias primas (como el caso del petróleo cuyo precio ha disminuido 48\% de enero de 2014 a junio de 2015, World Bank) por lo que Argentina no ha bajado en gran medida sus ingresos por exportación.

En lo referente a los destinos de sus exportaciones observamos que Brasil es su principal mercado de para sus exportaciones con el 20\% de participación, China el 7\% y Estados Unidos el 6\%, por lo que ante la recesión en Brasil y la disminución de materias primas por parte de China las épocas de auge de las exportaciones argentinas han quedado atrás, sin embargo la fortaleza de su mercado interno ha impedido que la economía caiga a niveles alarmantes. El comercio internacional representa el 29.5\% del PIB argentino, por lo que observamos que de los cuatro países seleccionados es el segundo con mayor independencia del sector externo, por debajo de Brasil.

Con el análisis del comercio exterior argentino se concluye que en el canal de transmisión de la inestabilidad financiera internacional hacia la devaluación de la moneda argentina el papel del comercio exterior no es central.

Pero de los cuatro países es el que acumula una mayor devaluación de su moneda $129 \%$ de 2011 a junio de 2015.

Para entender este fenómeno es importante observar el comportamiento histórico de las clases medias y altas de la sociedad argentina. Suelen mantener ahorros fuera de su país, por lo que ante la inestabilidad financiera, la corrida especulativa contra la moneda argentina es mayor, aun cuando sus cuentas de comercio exterior no se deterioren mucho. Ante esta situación el Banco Central Argentino ha intentado defender su moneda, lo que se observa en la caída en las reservas internacionales en $22 \%$ (ver cuadro 1). El caso argentino es particularmente complejo porque pareciera que la coyuntura de inestabilidad financiera internacional no le ha afectado en la magnitud de los otros países seleccionados. La gran devaluación del peso argentino ocurrió durante el año 2013, el tipo de cambio pasó de 4.9 a 8 pesos por dólar para febrero de 2014 . Para junio de 2015 registró 9 pesos por dólar. Es decir la coyuntura financiera 


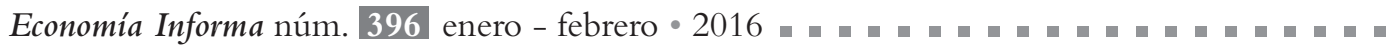

internacional de fines de 2014 y el primer semestre de 2015 parecen no haberle afectado en gran medida a Argentina dado que ellos realizaron un ajuste cambiario antes de la turbulencia.

Este ajuste cambiario se debió en gran medida a la acumulación de la inflación, debemos recordar que Argentina es el país con la mayor tasa inflacionaria entre los países seleccionados, ${ }^{1}$ por lo que la devaluación ha sido una manera de ajustar su economía. Sin embargo las cifras de crecimiento del PIB para el primer semestre de 2015 indican un alza del 2,3\% (Indec 2015), explicado en gran medida por las inversiones en construcción, infraestructura y por el fortalecimiento de su mercado interno. Por lo que se concluye que un posible incremento en la tasa de interés de Estados Unidos sí afectaría al peso argentino, pero dada la importancia que está tomando su mercado interno y la poca exposición del sistema financiero argentino a los mercados financieros internacionales, es posible, de mantenerse la fortaleza de su mercado interno, que irónicamente la moneda argentina sea de las monedas menos afectadas por un incremento en la turbulencia financiera.

\subsection{Brasil}

Brasil ha acumulado una devaluación de 86\% entre enero de 2011 y junio de 2015, la segunda más grande entre los países seleccionados. A diferencia de Argentina, Brasil sí ha sido víctima de la inestabilidad financiera de fines de 2014, la mayor devaluación ocurrió entre septiembre de 2014 y junio de 2015, el dólar pasó de 2.2 a 3.2 reales por dólar.

Dentro de la explicación de la devaluación del real brasileño encontramos una caída en los ingresos de sus exportaciones. Los principales productos para la exportación en Brasil son: el equipo de transporte, el mineral de hierro, la soya, el calzado, café y automóviles (CIA 2015). Es decir, hay un equilibrio entre manufactura y materias primas. Lo que atenúa la caída en los precios internacionales del petróleo, sin embargo, los principales destinos de sus exportaciones son China 18\%, Estados Unidos 12\%, Argentina 6\% y Holanda 6\% (2014). Por lo que ante la caída de la demanda de materia primas en China, Brasil ha ido a pique (Lyons y Kiernan 2015).

La importancia del comercio exterior en el PIB de Brasil es del 28.5\% (World Bank 2015), por lo que pareciera que la importancia de su mercado

No está claro el monto de la inflación en Argentina, de acuerdo con el Banco Mundial es del orden del $25 \%$, mientras que para el gobierno argentino es de alrededor del $12 \%$, en cualquier caso es la mayor entre los países seleccionados. 
interno puede sacar a flote a la economía tras las perspectivas negativas de los precios internacionales de las materias primas y en particular del petróleo, además el comercio exterior brasileño incluye manufacturas como el equipo de transporte, los automóviles y el calzado, por lo que pareciera que la importancia del comercio exterior en la explicación de la devaluación del 86\% de su moneda no es central.

A diferencia de Argentina la exposición del sistema financiero brasileño al mercado financiero mundial sí es mucha, y combinados los efectos de la inestabilidad financiera mundial por el posible incremento en la tasa de interés de la Fed, la caída generalizada de los rendimientos de los bonos corporativos en América Latina (Moody's 2015) y en particular la inestabilidad política, las protestas ciudadanas y los escándalos de corrupción en Brasil han coadyuvado a que la corrida especulativa en contra del real brasileño sea mayúscula.

Se observa claramente que el periodo de devaluación monetaria más agudo inició en septiembre de 2014, justo cuando la bolsa de valores de Sao Paulo presentó una clara tendencia a la baja, hasta llegar a un punto mínimo en enero de 2015, posteriormente se estancó y presentó una leve recuperación entre marzo y mayo de 2015, a partir de allí ha tenido un comportamiento en caída libre, a tal grado que la Bolsa Mexicana de Valores ha llegado a superar a la brasileña, siendo que históricamente Brasil cuenta con un mercado financiero más desarrollado que el mexicano, una de las consecuencias de este comportamiento lo refleja la devaluación del real en 50\% en la primera mitad de 2015.

La devaluación acumulada de $86 \%$ pareciera que augura una gran conflictividad en Brasil, sin embargo, el país cuenta con la estructura productiva más solida de Latinoamérica, cuenta con un mejor equilibrio de sus exportaciones entre materias primas y bienes manufacturados ( 9 de sus exportaciones se componen de materias primas, mientras que 6 son manufacturas), cuenta con mercados de exportación muy diversificados a diferencia de México (China, Estados Unidos, Argentina, CEE y Japón entre otros).

Por lo tanto, la importancia de su mercado interno en la composición del PIB es mucho mayor que el mercado mundial, alrededor del 75\% es mercado interno y los niveles de pobreza han bajado considerablemente fortaleciendo aún más a su mercado interno. Sin embargo, la productividad se mantiene estancada (al igual que en las demás economías latinoamericanas), por ejemplo el brasileño promedio cursa 7.5 años de escuela, mientras que en países como Corea es del doble (Universia Knowledge Wharton Pensylvania 2014), aunado a lo anterior, la inversión se ha estancado en 18\% del PIB lo que ha generado una falta de mejora en su infraestructura. 
Como se observa la estructura productiva de Brasil es la más sólida de Latinoamérica pero no escapa a los problemas de atraso en infraestructura y productividad lo que vuelve vulnerable a la economía, pero quizás el problema más agudo lo constituye la coyuntura política en la que se encuentra, los escándalos de corrupción en Petrobras y la presión de la clase empresarial hacia una política económica más favorable para ellos y que vele menos por la redistribución del ingreso se conjuntan en un segundo periodo presidencial de Rousseff muy complicado y expuesto al escrutinio internacional ante las justas deportivas mundiales de 2014 y 2016. Por ello se prevé una crisis de liderazgo en la economía brasileña en la que los escenarios son inciertos, podría recomponer su popularidad el gobierno y capitalizar sus avances industriales y de fortalecimiento en el mercado interno pasando hacia una nueva fase de incremento de la productividad y de la inversión aún a costa de un crecimiento del PIB modesto en el corto plazo; o es posible que veamos una transformación de la economía hacia elevar las ganancias en el corto plazo mediante controles salariales e inflacionarios, mayores ventajas para el capital financiero internacional pero generando una mayor vulnerabilidad económica en el mediano y largo plazo.

\subsection{Colombia}

Colombia ha sufrido una devaluación de su moneda del orden de 35\%. El peso colombiano se había mantenido estable entre 2011 y el primer semestre de 2014, pero a partir de agosto de ese año, pasó de 1,800 pesos colombianos por dólar a 2,500, es decir la devaluación se ha concentrado en el periodo de mayor volatilidad financiera segundo semestre de 2014 y primer semestre de 2015.

La perspectiva para Colombia es complicada en lo económico porque sus principales productos de exportación son: el petróleo (sexto productor de América), el carbón, las esmeraldas, el níquel, las flores, el plátano y el café (tercer productor mundial CIA 2015). Es decir, básicamente materias primas, por ello, ante una caída en la demanda mundial, los precios de las materias primas producidas por Colombia han bajado y con ello sus ingresos por exportación. Por lo que el panorama económico se observa adverso dado el bajo precio de las materias primas en el mercado mundial. Además Colombia depende mucho de su comercio exterior, el 37.5\% de su PIB proviene de sus importaciones y exportaciones (el segundo de la región sólo detrás de México), por lo que ante un deterioro de los términos de intercambio en el mer- 
cado mundial, sus motores endógeno no se observan tan sólidos como el caso brasileño o el argentino.

Los principales destinos de sus exportaciones son Estados Unidos 26\%, China $11 \%$ y Panamá $7 \%$, por lo que ante un posible crecimiento menor de las economías estadounidense y china, el mercado de sus exportaciones se verá severamente golpeado.

Sin embargo, la caída de los ingresos por exportaciones no ha impactado a sus reservas internacionales, entre 2011 y el primer semestre de 2015 sus reservas han crecido en $63 \%$, lo que plantea la poca vinculación entre la devaluación de su moneda y una pérdida de reservas internacionales. Por otro lado se observa la poca injerencia de su Banco Central para mantener el tipo de cambio, en todo caso el margen de maniobra para defender el peso colombiano es amplio al tener un importante incremento en las reservas internacionales (ver cuadro 1 ).

De manera similar con Brasil la devaluación de la moneda colombiana tiene una explicación por la exposición de su economía a la alta volatilidad de los mercados financieros internacionales, la suma de elementos diversos como la caída del precio internacional del petróleo (su principal producto de exportación), la consecuente caída de la Inversión Extranjera Directa (IED), porque la mayoría de ésta se dirige al sector petrolero, el incremento de la deuda pública y del déficit de cuenta corriente (30\% del PIB), el incremento del riesgo país por la inseguridad por el problema con las FARC y sobre todo el entrono internacional de alta volatilidad y aversión al riesgo, han generado una fuerte corrida especulativa en contra del peso colombiano.

La estructura productiva de Colombia no parece tan sólida como la brasileña, la principal industria manufacturera es la textil y la química, por lo que ante el entorno recesivo de la demanda mundial, los motores endógenos de Colombia no se muestran sólidos para sacar a la economía adelante, por lo que un incremento en el precio internacional de las materias primas resulta central para el desempeño de la economía.

En lo político, a diferencia de Brasil y México, el Presidente Juan Manuel Santos (proveniente de la oligarquía empresarial) goza de un alto grado de legitimidad, por lo que pudiera tener apoyo para aplicar medidas audaces ante un posible escenario de emergencia económica. 


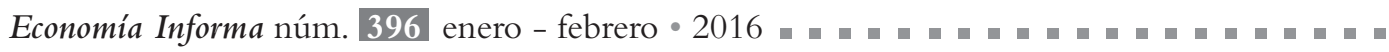

\subsection{México}

El caso mexicano es totalmente distinto al de las demás naciones latinoamericanas, observando las cifras del comercio exterior podríamos concluir que es la economía más sólida de América Latina, porque cuenta, por mucho, con la mayor diversificación en sus exportaciones, siendo sus principales productos: el petróleo crudo, los automóviles, las autopartes, partes y accesorios de tractores, computadoras, televisores, teléfonos, cables coaxiales y minerales (carbón, plata, oro y piedras preciosas). Es decir pareciera que la industria manufacturera en México es sólida y muy diversificada, sin embargo, si analizamos con detalle encontramos que la mayor parte de la manufactura que se exporta, e incluso que se produce es por la industria maquiladora, es decir en México se produce en realidad escaso valor agregado, las empresas, la tecnología y las ganancias son extranjeras generando limitadas cadenas de valor y propiciando un importante grado de dependencia hacia la economía de Estados Unidos. Por lo tanto las exportaciones que sí dependen de los encadenamientos de valor mexicanos son: el petróleo y la minería. Por ello ante la caída de los precios internacionales de las materias primas, la economía mexicana está sufriendo porque sigue siendo altamente dependiente del petróleo aunque a simple vista pareciera que no.

Prueba de ello son dos importantes anuncios: el Banco Central (Banxico) recortó su expectativa de crecimiento a un margen entre 1.7 y $2.5 \%$ para este año; y la Secretaría de Hacienda anunció un fuerte programa de recorte en el gasto público por la caída de los ingresos petroleros.

En este tenor la perspectiva de los ingresos por exportaciones es sombría para México, el 80\% de sus exportaciones se dirigen a Estados Unidos y en realidad son producto de la industria maquiladora en su mayoría, lo que implica que la sincronización del ciclo económico entre México y Estados Unidos es muy profundo, si la empresa estadounidense detiene sus inversiones, entonces la industria maquiladora en México se detiene y con ello una buena parte de la actividad económica y de las exportaciones. Un dato resulta muy representativo de la fragilidad de la estructura productiva en México: el 66.4\% de su PIB proviene de su comercio exterior, y de ese 66\%, las exportaciones hacia Estados Unidos representan el 80\% y las importaciones procedentes de Estados Unidos representan el 50\% (Comtrade 2015).

Ante la situación económica incierta en Estados Unidos, los efectos económicos sobre México de una nueva recesión pueden ser mayúsculos.

En este sentido la devaluación del peso mexicano de $28 \%$ entre enero de 2011 y junio de 2015 es el resultado de la debilidad estructural de la economía 
mexicana, del grado de dependencia hacia la economía de Estados Unidos, la cual se encuentra en recesión, de la caída de los ingresos por exportaciones y de la corrida especulativa en contra del peso. El margen de maniobra del Banxico para defender a la moneda mexicana aún es amplio entre enero de 2011 y junio de 2015 las reservas internacionales han crecido en 109\%, este dato se explica en gran medida por el diferencial de tasas de interés entre México y Estados Unidos, mientras en este último país es de cerca de $0 \%$ en México es de alrededor de 3\%, lo que genera importantes entradas de capital financiero en México aunque se encarezca el costo de las inversiones y la economía nacional no crezca.

Como se observa el gobierno mexicano está firmemente encadenado al accionar de la economía estadounidense y no parece intentar cambiar, la legitimidad del presidente es muy baja, y se ha acentuado a partir de la desaparición de los estudiantes en el estado de Guerrero, pero aún le restan 3 años a su periodo presidencial, por lo que no se observa en el horizonte vientos de cambio en la política económica de México, más bien la apuesta continúa en una mejora en la economía estadounidense que genere derramas sobre México. Pero aún esta apuesta parece riesgosa si consideramos la devaluación de $28 \%$ del peso mexicano provocado por la inestabilidad que genera la probabilidad de incremento en la tasa de interés de Estados Unidos. Si la economía estadounidense repunta, no habrá razones para mantener la tasa de interés en $0 \%$, por lo que si bien ante un mejoramiento de la economía estadounidense el sector exportador mexicano podría mejorar, la moneda mexicana podría devaluarse aún más por efecto de la disminución en el diferencial de tasas de interés entre México y Estados Unidos. Este proceso afectaría en gran medida al ya muy golpeado mercado interno, generando un incremento en la inflación en México dado el alto contenido de las importaciones en el ciclo de reproducción económica mexicano.

Pero es posible también otro escenario, que la inestabilidad financiera descrita en la primera parte de este texto termine por generar una nueva caída del sector financiero de Estados Unidos, lo que seguramente arrastraría hacia abajo aún más a la economía mexicana por los canales ya descritos (caída de la demanda de nuestras exportaciones, caída en la IED, caída en la industria maquiladora y en las remesas). Obligando al nuevo gobierno en 2018 a replantear la estructura de la acumulación en México volteando hacia la diversificación del comercio internacional (tarea nada sencilla) y hacia el fortalecimiento del mercado interno, para tal efecto el grado de legitimidad del siguiente presidente es crucial para poder emprender cambios tan audaces pero necesarios. 
Economía Informa núm. 396 enero - febrero • 2016

A continuación se agrupan los principales rubros de los cuatro países seleccionados en un cuadro resumen.

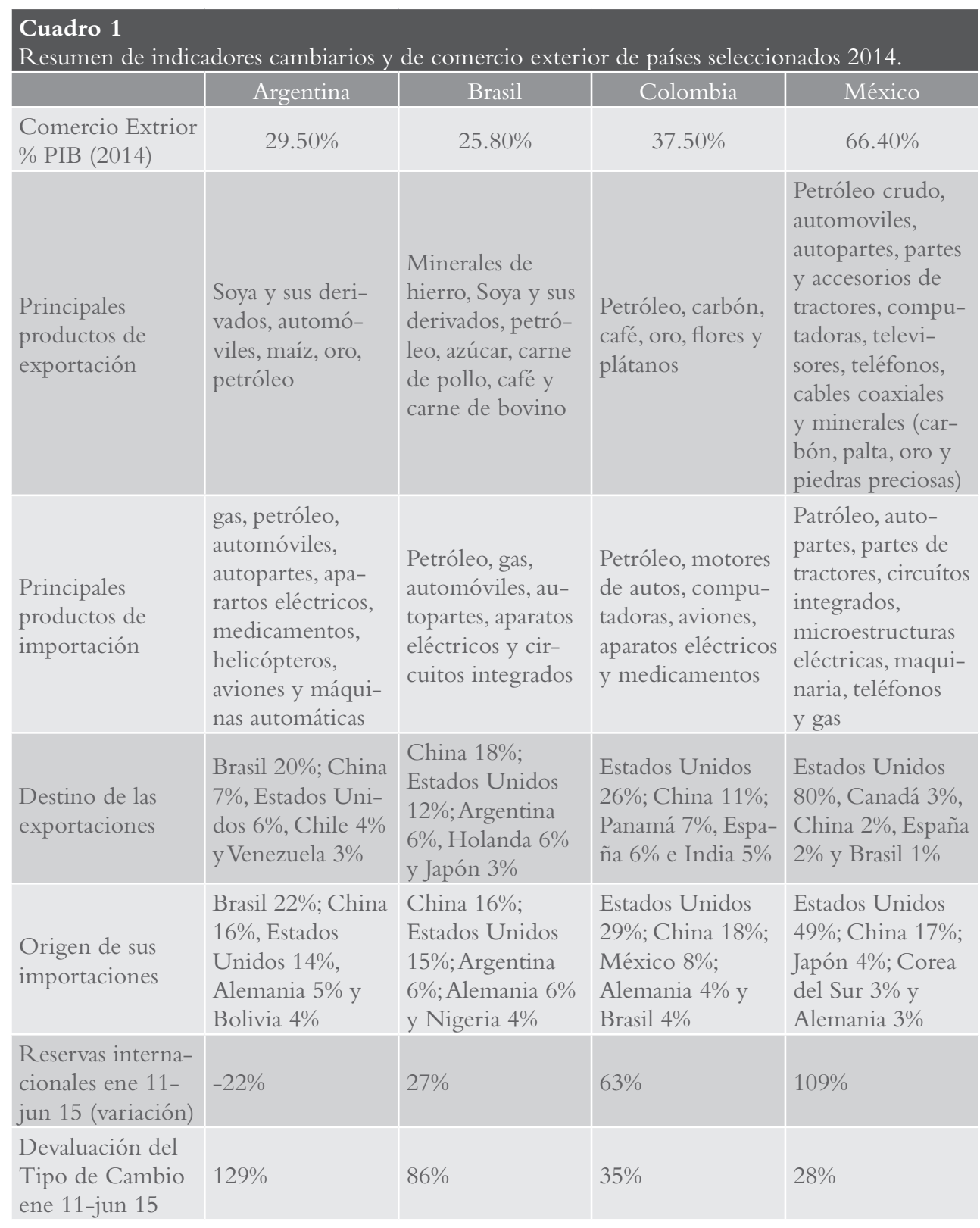

Fuente: World Bank, Comtrade, IMF, Bancos Centrales de Argentina, Colombia, Brasil y México. 


\section{Conclusiones}

Los escenarios a futuro que se observan en la economía mundial son de un proceso irreversible de cambios en el ámbito monetario y financiero que reflejan las transformaciones en le hegemonía mundial (Dabat y Leal 2013). Con una economía estadounidense tambaleante y la falta de un nuevo país que tome la batuta de la hegemonía. Esta falta de clara hegemonía mundial se traduce en un desorden monetario y financiero a nivel mundial que genera alta volatilidad en los mercados de bonos.

Para enfrentar esta situación Estados Unidos ha bajado la tasa de interés de los bonos del tesoro provocando un incremento de la liquidez mundial nunca antes vista, lo que contribuye aún más con la inestabilidad financiera.

En la economía real, el ciclo ascendente en el precio de las commodities ha llegado a su fin, por lo que el precio del petróleo y de otras materias primas se ha derrumbado, este proceso ha beneficiado a la economía mundial en su conjunto porque dada la enorme liquidez mundial, no estamos sufriendo de alta inflación como en los años setenta, sin embargo, para las economías exportadoras de commodities como es el caso de los cuatro países seleccionados resulta en un grave deterioro de sus términos de intercambio.

La carencia de un escenario de altos ingresos por exportaciones, más la inestabilidad financiera y la falta de un núcleo endógeno de acumulación basado en alta productividad ha arrastrado a los cuatro países seleccionados a un deterioro de su economía y a niveles importantes de devaluación de sus monedas nacionales.

En el análisis detallado de los cuatro países de la región observamos la clara falta de vinculación de México con el resto de países de América Latina. Brasil, Argentina y Colombia (en menor medida) se encuentran más integrados con el cono sur, pero también con Europa y con China.

La estructura económica más sólida de los cuatro países es la de Brasil. Cuenta con una industria manufacturera más sólida e independiente, pero se encuentra en una coyuntura mundial muy compleja que su salida dependerá en mucho del precio internacional de las commodities, pero también de la estabilidad política que pueda lograr en el corto plazo. En el caso de Argentina se observa un entorno político complicado, pero menos adverso que en Brasil con una estructura económica menos fuerte que la brasileña, pero con un momento económico más favorable: ingresos importantes por la exportación de soya y transición hacia un más sólido mercado interno. Colombia cuenta con una importante inestabilidad por conflictos añejos con la guerrilla 


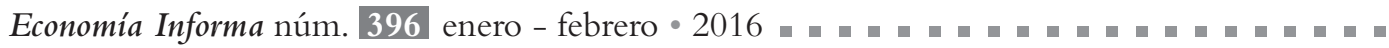

y el narcotráfico, la estructura económica más débil de los países seleccionados, (medido a partir de su industria manufacturera y su mercado interno). Depende mucho más que los demás países del precio de las commodities, por lo que de no emprender ajustes importantes en su economía será presa de la inestabilidad financiera mundial que no tiene visos de fin.

Por su parte México cuenta con una estructura productiva muy compleja en el que el mercado interno es débil por la alta desigualdad del ingreso (Esquivel 2015), una industria manufacturera muy golpeada por la indiscriminada apertura comercial, una participación muy grande de la economía informal, entre otros rubros del narcotráfico (lo que hace más difícil el análisis), una dependencia mayúscula hacia la economía estadounidense (la más grande de la región), un muy pobre nivel de legitimidad de su gobernante y una élite empresarial muy ligada al sector externo. Este conjunto de elementos convierte a México en la nación con menos probabilidades de emprender cambios drásticos en su economía en el corto plazo. Es el país más expuesto al comportamiento de la economía estadounidense, pero con una gran paradoja si Estados Unidos se recupera e incrementa la tasa de interés, México se verá la disyuntiva de sufrir una mayor devaluación de su moneda, o incrementar la tasa de interés y con ello golpear aún más a la inversión productiva doméstica y al mercado interno. Pareciera que sólo un drástico cambio en la correlación de fuerzas políticas en el país podría llevar a México a emprender vías de acumulación más autónomas.

\section{Bibliografía}

Banco Central de Colombia (2015) Sitio web: http://www.banrep.gov.co/es/-estadisticas, consultado el 11 de septiembre de 2015.

Banco Central de la República Argentina (2015) sitio web: http://www.bcra.gov. ar/Estadisticas/estser030100.asp, consultado el 3 de junio de 2015.

Banco Central Do Brasil (2015) sitio web : http://www.bcb.gov.br/?serietemp, consultado el 19 de junio de 2015.

Banxico (2015) sitio web: http://www.bancodemexico.gob.mx/estadisticas/index. html, consultado el 10 de agosto de 2015.

Barr Colin (2015) "El mercado de bonos es más grande y frágil”, The Wal Street Journal Americas, Estados Unidos, 22 de septiembre de 2015.

Board of Governors of the Federal Reserve System (2015), United States of America sitio web: http://www.federalreserve.gov/econresdata/default.htm, consultado el 2/10/2015. 
Bureau of Economic Analysis (2015), sitio web: http://www.bea.gov/itable/index. cfm, consultado el 30/09/2015.

Cherney Mike (2015) "Los bonos corporativos alertan sobre la economía de EE.UU." The Wall Street Journal Americas, Estados Unidos, 22 de septiembre de 2015.

CIA (2015) World Fact Book, sitio web: https://www.cia.gov/, consultado el 7/09/2015.

Comtrade (2015) sitio web: http://comtrade.un.org/data/, consultado el 23/09/2015.

Dabat, Alejandro y Leal Paulo (2013) "Declinación de Estados Unidos: contexto histórico mundial", Problemas del Desarrollo, 44, Número 174, IIEc UNAM.

Esquivel, Gerardo (2015) "Desigualdad Extrema en México", Oxfam, México.

IMF (2015) sitio web: http://www.imf.org/data, consultado el 1 de octubre de 2015.

Instituto Nacional de Estadística y Censos INDEC (2015) Ministerio de Economía y Finanzas Públicas República argentina.

Leal Paulo (2015) La estructura económica como determinante de la relación Estados Unidos - México, (1940-2013), Tesis doctoral en economía, Instituto de Investigaciones Económicas, UNAM, México.

Lyons John y Kiernan Paul (2015) “How Brazil's China-Driven Commodities Boom Went Bust", The Wall Street Journal, 27 de agosto de 2015.

Moody's Investor's Service (2015) "Cae emisión de bonos corporativos en América Latina”, sitio web https://www.moodys.com/Pages/atc002.aspx, consultado el 30 de abril de 2015.

Universia Knowledge Wharton Pensylvania (2014) "Brasil ante el reto de aumentar la productividad", sitio web: https://www.knowledgeatwharton.com.es/, consultado 20 de septiembre de 2015.

World Bank (2015) http://data.worldbank.org/. Consultado 3 de junio de 2015. 